\title{
The Case for Discrete Energy Levels of a Black Hole
}

\author{
JACOB D. BEKENSTEIN" \\ Racah Institute of Physics, The Hebrew University of Jerusalem \\ Givat Ram, Jerusalem 91904 ISRAEL
}

\begin{abstract}
The adiabatic invariant nature of black hole horizon area in classical gravity suggests that in quantum theory the corresponding operator has a discrete spectrum. I here develop further an algebraic approach to black hole quantization which starts from very elementary assumptions, and proceeds by exploiting symmetry. It predicts a uniformly spaced area spectrum for all charges and angular momenta. Area eigenvalues are degenerate; correspondence with black hole entropy then dictates a precise value for the interval between eigenvalues.
\end{abstract}

\section{Introduction}

To many the term "black hole" means a macroscopically large object, the engine behind the flickering X-ray sources in the Galaxy, the quasars and other active galactic nuclei. Talking about the discrete energy spectrum of a black hole, a patently quantum issue, must seem incredibly pedantic: why not just use classical physics ? But, of course, quantum dynamics is the underlying dynamics of the world, and the simplicity of black holes just begs us to use them to learn about quantum gravity. The nature of the energy spectrum of a black hole is about the simplest question that can be asked in this context. Here I would like to approach the issue from a unconventional angle.

One usually discusses an energy spectrum for a collection of stationary states. Classically stationary states of a black hole are described by the Kerr-Newman (KN) joint solution to the Einstein and Maxwell equations [1]. Its parameters, mass $M$, electric charge $Q$ and angular momentum $\mathbf{J}$, can all be inferred from the asymptotic behavior of the electromagnetic field and geometry, and are thus observables in a true sense. The special case $\mathbf{J}=0$ of $\mathrm{KN}$ is called the Reissner-Nordström black hole; that with $\mathbf{J}=0$ and $Q=0$ corresponds to the original Schwarzschild black hole. For a quarter century it was widely accepted that the KN black holes account for all stationary black states within general relativity and a number of similar gravity theories, at least if one overlooks trivial extensions of KN like including Dirac monopole number alongside electric charge. This "no hair principle" was overturned in the 1990's with the appearance of a number of new black hole parameters and properties: skyrmion

\footnotetext{
${ }^{1}$ email: bekenste@vms.huji.ac.il
} 
number, nonabelian magnetic monopole, color, etc. These complications are irrelevant to our task. I will here pretend that $M, Q$ and $\mathbf{J}$ are the only parameters of stationary black hole states.

\section{Adiabatic Invariance and Black Hole Mass Quan- tization}

Like any observable of a KN black hole, the area of its event horizon (boundary) can be expressed as a function of $M, Q$ and $\mathbf{J}$ :

$$
A=4 \pi\left[\left(M+\sqrt{M^{2}-Q^{2}-\mathbf{J}^{2} / M^{2}}\right)^{2}+\mathbf{J}^{2} / M^{2}\right],
$$

where I am using units with $G=c=1$ in which $\sqrt{ } \hbar$ stands for either the Planck's length or the Planck mass. Any departure from stationarity is likely to involve an increase in horizon area [2]. But when slow changes of a black hole occur, $A$ behaves as an adiabatic invariant [3, 4]. Recall that an adiabatic invariant is a quantity which changes especially slowly in the wake of a slow change of the system's parameter. For an harmonic oscillator whose cord is gradually extended on a timescale well exceeding its period, the ratio of the oscillator's energy to its frequency is constant to exponential order, and hence an adiabatic invariant.

Lower slowly (adiabatically) a point charge $\varepsilon$ down to the horizon of a ReissnerNordström black hole of mass $M$ and charge $Q \gg \varepsilon$, and then allow it to be assimilated by the hole. The black hole obviously experiences changes: $\delta Q=\varepsilon$ and $\delta M=\varepsilon \Phi$, where $\Phi$ is the electrostatic potential at the horizon (the particle's rest energy is entirely redshifted away). From the KN solution we have

$$
\Phi=\frac{Q}{M+\left(M^{2}-Q^{2}\right)^{1 / 2}}
$$

The increment of the horizon's area, Eq. (1), is

$$
\delta A=(\delta M-\Phi \delta Q) \Theta^{-1} ; \quad \Theta \equiv \frac{1}{2}\left(M^{2}-Q^{2}\right)^{1 / 2} A^{-1}
$$

which evidently vanishes for $\delta Q=\varepsilon$ and $\delta M=\varepsilon \Phi$. Thus to $O(\delta Q)$ the area is unchanged: classically $A$ and all smooth functions of it are adiabatic invariants. This example can be extended to the Schwarzschild and generic Kerr-Newman black holes [5]. Several other examples demonstrating adiabatic invariance of horizon area under electromagnetic or scalar field perturbations have been presented by Mayo [4. The adiabatic invariant nature of $A$ is useful in at least one astrophysical context [6], and seems to extend to quantum gravity [7].

The basic conclusion of our example is unchanged when the quantum mechanics of the charge is taken into account. A simple calculation [8] shows that although there is now a change in horizon area, $\delta A \geq \xi \hbar$ with $\xi=O(1)$ and independent of 
the particle's charge and mass, and of type and scale of black hole, the minimum area increase is, again, of second order, and thus negligible. The adiabatic invariant status of $A$ is sustained.

Ehrenfest's principle [9] of the old quantum mechanics, a classical adiabatic invariant corresponds to a quantum observable with a discrete spectrum, now implies that the operator $\hat{A}$ in the quantum theory (we denote operators by carets), which corresponds to classical horizon area, is expected to have a discrete spectrum: $\left\{a_{1}, a_{2}, \cdots a_{n} \cdots\right\}$. We may even guess from the fact that introducing a quantum particle into a KN black hole carries a minimal "cost" $\delta A=\xi \hbar$ which does not depend on how big the black hole is, that the spacing between area eigenvalues of the $\mathrm{KN}$ area spectrum is a uniform one. This way the smallest jump in area corresponds to a transition between neighboring "area levels".

Such a simple structure of the spectrum of area warrants considering $\hat{A}$ alongside the charge observable $\hat{Q}$ and the angular momentum one $\hat{\mathbf{J}}$ as the fundamental observables of a black hole. From this point of view black hole mass $\hat{M}$ is a secondary observable. We may guess it is given by the Christodoulou-Ruffini formula [10 (obtained by inverting (11))

$$
M=\left[\frac{A}{16 \pi}\left(1+\frac{4 \pi Q^{2}}{A}\right)^{2}+\frac{4 \pi \mathbf{J}^{2}}{A}\right]^{1 / 2}
$$

and replacing $A \rightarrow \hat{A}$, etc. This together with the equispaced area spectrum implies the mass spectrum

$$
M \sim \sqrt{\hbar n} \quad n=1,2, \cdots
$$

for the quantum Schwarzschild black hole [11, 12, 13.

As we shall see, such mass spectrum is inevitably highly degenerate. It is also strongly at variance with Hawking's celebrated semiclassical result for the spectrum radiated by a black hole - a continuum with roughly Planckian shape. Our prediction tells us that quanta emitted as a result of the black hole jumping one or a few steps of the $\sqrt{ } n$ spectrum should have frequencies which are quite accurately multiples of a frequency $\sim 1 / M$, of order of the peak frequency in Hawking's spectrum. What does full blown quantum gravity have to say about this?

As summarized in my 2001 Erice lectures [8], a number of workers employing canonical quantization of gravity have obtained a mass spectrum of this form for the Schwarzschild black hole, but with no consensus as to the exact numerical coefficient [14]. The story is somewhat similar for charged and rotating black holes [15, (7). Some canonical gravity calculations give a not uniformly spaced spectrum [16]. The same is found by loop quantum gravity methods [17], but there are two claims that an equispaced area spectrum is consistent with loop gravity after all [18]. In string theory an extreme black hole (a BPS state) has horizon area which is quantized as the square root of a product of integers (the charges). This is in the spirit of the finding here, but the rule does not extend to the nonextreme black holes which is said to posses a continuum mass spectrum. 
If no errors are involved, then the various approaches must be elucidating the black hole spectrum at various levels of accuracy. For example, it may be that (5) gives the pristine spectrum before any level broadening by radiative effects or any degeneracy breaking by perturbations is effective, while the string result describes the situation after all perturbations are taken into effect. It would be nice to find out for sure, and by the simplest logic, what the pristine spectrum looks like. In what follows I describe a purely algebraic approach to the black hole area spectrum which, to my mind, clarifies this question.

\section{Black Hole Algebra}

Like any other quantum system, the quantum black hole should exhibit the usual angular momentum spectrum, Spect $\left(\hat{\mathbf{J}}^{2}\right)=\left\{j(j+1) \hbar^{2} \mid j=0, \frac{1}{2}, 1, \cdots\right\}$, Spect $\left(\hat{J}_{z}\right)=$ $\{m \hbar \mid m=-j,-(j-1), \cdots, j\}$. This is known to follow from the algebra of angular momentum

$$
\hat{\mathbf{J}} \times \hat{\mathbf{J}}=\imath \hbar \hat{\mathbf{J}}
$$

which we adopt for the black hole. We likewise expect on intuitive grounds that there exist one-black hole states, denoted by $|n j m q s\rangle$, each of which can be specified simultaneously by $j$ and $m$ as well as the eigenvalue of charge $\hat{Q},\{q e \mid q=0, \pm 1, \pm 2, \cdots\}$ (e denotes the elementary charge), that of $\hat{A},\left\{a_{n} \mid n=1,2,3, \cdots\right\}$, and an additional quantum number, $s$, which distinguishes between states with common sets $\{n q j m\}$; $s=1,2, \cdots, g_{n} ; g_{n}$ is the degeneracy of the said states. Evidently this requires at the very minimum that

$$
[\hat{\mathbf{J}}, \hat{Q}]=0 ; \quad[\hat{\mathbf{J}}, \hat{A}]=0 ; \quad[\hat{Q}, \hat{A}]=0 .
$$

The first two conditions are justified because both charge and area should be invariant under rotations. The last follows if area is gauge invariant (charge is the generator of a global gauge transformations). Gour [19] has succeeded in defining an operator whose eigenvalues are $s$; however, he presupposes that the spectrum of $\hat{A}$ is equispaced. Since showing this is one of our goals we choose a different approach. Finally, we define the black hole vacuum $|\mathrm{vac}\rangle$ (assumed unique) as the state with vanishing area, $j=0$ and $q=0$.

For every basis state define an operator $\hat{R}_{n j m q s}$ such that $|n j m q s\rangle=\hat{R}_{n j m q s}|\mathrm{vac}\rangle$. This leaves a lot of freedom in $\hat{R}_{n j m q s}$ which we shall exploit to our convenience forthwith. To imbue the algebra of $\hat{\mathbf{J}}, \hat{Q}, \hat{A}, \hat{I}$ (unity operator) and $\hat{R}_{n j m q s}$ with some nontrivial content, we shall assume that it is closed and linear. Closure is assumed for simplicity: no new operators arise from commuting those already present. Linearity (the commutator of any two operators in the algebra is a linear combination of some of the operators in the algebra) is a strong assumption because one might inquire what singles out $\hat{A}$ as opposed to, say, $\sqrt{ } \hat{A}$ as the operator in terms of which the algebra is linear. Our ultimate justification is that the algebra, as stated and in no 
other form, makes $\hat{A}$ an additive quantity for systems of several black holes [8], just as are $\hat{Q}$ and $\hat{J}_{z}$, and just as intuition demands.

By definition $\hat{Q}|n j m q s\rangle=q e|n j m q s\rangle$ so that

$$
\exp (\imath \chi \hat{Q}) \hat{R}_{n j m q s}|\operatorname{vac}\rangle=\exp (\imath \chi q e) \hat{R}_{n j m q s}|\operatorname{vac}\rangle .
$$

Thus $\exp (\imath \chi \hat{Q})$ performs a global gauge transformation on the basis states. One thus suspects that the same operator transforms all the $\hat{R}$ operators as

$$
\exp (\imath \chi \hat{Q}) \hat{R}_{n j m q s} \exp (-\imath \chi \hat{Q})=\exp (\imath \chi q e) \hat{R}_{n j m q s}
$$

because operating with this last on $|v a c\rangle$ reproduces Eq. (8). To $O(\chi)$ this last equation gives another basic commutator

$$
\left[\hat{Q}, \hat{R}_{n j m q s}\right]=q e \hat{R}_{n j m q s}
$$

Passing on we note that under rotations $|\mathrm{vac}\rangle$ must be invariant but $\hat{R}_{n j m q s}|\mathrm{vac}\rangle$ must transform like the corresponding spherical harmonics $Y_{j m}$ (or spinorial harmonic if $j$ is half-integer). Thus $\hat{R}_{n j m q s}$ must be an irreducible spherical tensor operator of rank $j$ [20]. This entails the commutators

$$
\begin{aligned}
& {\left[\hat{J}_{z}, \hat{R}_{n j m q s}\right]=m \hbar \hat{R}_{n j m q s}} \\
& {\left[\hat{J}_{ \pm}, \hat{R}_{n j m q s}\right]=\sqrt{j(j+1)-m(m \pm 1)} \hbar \hat{R}_{n j, m \pm 1, q s}}
\end{aligned}
$$

where $J_{ \pm}=J_{x} \pm \imath J_{y}$. How does $\hat{A}$ commute with the $\hat{R}_{n j m q s}$ ? We start with Jacobi's identity $\left[\hat{A},\left[\hat{V}, \hat{R}_{n j m q s}\right]\right]+\left[\hat{V},\left[\hat{R}_{n j m q s}, \hat{A}\right]\right]+\left[\hat{R}_{n j m q s},[\hat{A}, \hat{V}]\right]=0$ with $\hat{V} \rightarrow\left\{\hat{J}_{z}, \hat{J}_{ \pm}, \hat{Q}\right\}$ to obtain

$$
\begin{aligned}
& {\left[\hat{Q},\left[\hat{A}, \hat{R}_{n j m q s}\right]\right]=q e\left[\hat{A}, \hat{R}_{n j m q s}\right]} \\
& {\left[\hat{J}_{z},\left[\hat{A}, \hat{R}_{n j m q s}\right]=m \hbar\left[\hat{A}, \hat{R}_{n j m q s}\right]\right.} \\
& {\left[\hat{J}_{ \pm},\left[\hat{A}, \hat{R}_{n j m q s}\right]\right]=\sqrt{j(j+1)-m(m \pm 1)} \hbar\left[\hat{A}, \hat{R}_{n j, m \pm 1, q s}\right] .}
\end{aligned}
$$

Note that these commutators and those in (7) are invariant under $\hat{A} \rightarrow \hat{A}+$ const. $\times \hat{I}$. To eliminate the freedom in $\hat{A}$ we demand that $\hat{A}|\mathrm{vac}\rangle=0$. Further, we note that the commutators (13-15) parallel those of $\hat{R}_{n j m q s}$ alone with $\hat{\mathbf{J}}$ and $\hat{Q}$ meaning that $\left[\hat{A}, \hat{R}_{n j m q s}\right]$ transforms under rotations and gauge transformations exactly like $\hat{R}_{n j m q s}$. In view of this, linearity and closure tell us that $\left(\hat{J}_{0} \equiv \hat{J}_{z}, \hat{J}_{ \pm 1} \equiv \hat{J}_{ \pm}\right)$

$$
\left[\hat{A}, \hat{R}_{n j m q s}\right]=\sum_{n^{\prime} s^{\prime}} h_{n s}^{n^{\prime} s^{\prime}} \hat{R}_{n^{\prime} j m q s^{\prime}}+\delta_{q}^{0}\left[\delta_{j}^{0}\left(C_{n s} \hat{I}+D_{n s} \hat{Q}+E_{n s} \hat{A}\right)+\delta_{j}^{1} F_{n s} \hat{J}_{m}\right]
$$

Here the matrix $h_{n s}^{n^{\prime} s^{\prime}}$ could depend on $q, j$ and $m$, but the various $C_{n s}, D_{n s}, E_{n s}$ and $F_{n s}$ are just complex constants. We have not included a $\hat{R}_{n j m q s}$ in the r.h.s. with $j$ and 
$q$ other than seen in the l.h.s. since such operator would transform under rotations and gauge transformations differently than the l.h.s. For like reason the $\hat{I}, \hat{Q}$ and $\hat{A}$, all gauge and rotationally invariant, occur in the r.h.s. only in conjunction with $R_{n 000 s}$ in the l.h.s., and the (spherical) components of $\hat{\mathbf{J}}$, which transform like an irreducible tensor of rank one, but are invariant under gauge transformations, occur in the r.h.s. only in conjunction with $R_{n 1 m 0 s}$ in the l.h.s.

When we operate on $|\operatorname{vac}\rangle$ with Eq. (16), $\hat{\mathbf{J}}, \hat{Q}$ and $\hat{A}$ kill this state, $\hat{I}$ preserves it, while the various $\hat{R}_{n j m q s}$ create one-black hole states. But one cannot represent the vacuum as a linear superposition of $|n j m q s\rangle$. Thus we must set $C_{n s}=0$. Similarly, $|n j m q s\rangle$ with different $n$ have to be orthogonal (because $\hat{A}$ is hermitian), and those with different $s$ (even if $n, j, m$ and $q$ are common) can be made orthogonal by Schmidt orthogonalization. Therefore we must have $h_{n s}^{n^{\prime} s^{\prime}}=k_{n s} \delta_{n}^{n^{\prime}} \delta_{s}^{s^{\prime}}$. We are thus left with

$$
\left[\hat{A}, \hat{R}_{n j m q s}\right]=k_{n s} \hat{R}_{n j m q s}+\delta_{q}^{0}\left[\delta_{j}^{0}\left(D_{n s} \hat{Q}+E_{n s} \hat{A}\right)+\delta_{j}^{1} F_{n s} \hat{J}_{m}\right]
$$

Operating with this on the vacuum shows that necessarily $k_{n s}=a_{n}$. Let us introduce new black hole creation operators

$$
\hat{\mathcal{R}}_{n j m q s} \equiv \hat{R}_{n j m q s}+\left(a_{n}\right)^{-1} \delta_{q}^{0}\left[\delta_{j}^{0}\left(D_{n s} \hat{Q}+E_{n s} \hat{A}\right)+\delta_{j}^{1} F_{n s} \hat{J}_{m}\right]
$$

Obviously the algebra of $\hat{\mathbf{J}}, \hat{Q}, \hat{A}, \hat{I}$ and $\hat{\mathcal{R}}_{n j m q s}$ is still linear and closed, and $\hat{\mathcal{R}}_{n j m q s}$ creates the same state as $\hat{R}_{n j m q s}$. The commutators (10)-(12) and (17) are replaced by

$$
\begin{aligned}
{\left[\hat{Q}, \hat{\mathcal{R}}_{n j m q s}\right] } & =q e \hat{\mathcal{R}}_{n j m q s} \\
{\left[\hat{J}_{z}, \hat{\mathcal{R}}_{n j m q s}\right] } & =m \hbar \hat{\mathcal{R}}_{n j m q s}-\left(m \hbar / a_{n}\right) \delta_{q}^{0} \delta_{j}^{1} F_{n s} \hat{J}_{m} \\
{\left[\hat{J}_{ \pm}, \hat{\mathcal{R}}_{n j m q s}\right] } & =\sqrt{j(j+1)-m(m \pm 1)} \hbar \hat{\mathcal{R}}_{n j, m \pm 1, q s} \\
& -\left(\sqrt{2-m(m \pm 1)} \hbar / a_{n}\right) \delta_{q}^{0} \delta_{j}^{1} F_{n s} \hat{J}_{m \pm 1} \\
{\left[\hat{A}, \hat{\mathcal{R}}_{n j m q s}\right] } & =a_{n} \hat{\mathcal{R}}_{n j m q s}
\end{aligned}
$$

The first three equations include contributions which vanish due to factors of the form $q \delta_{q}^{0}, \delta_{j}^{0} \sqrt{j(j+1)-m(m \pm 1)}$, etc.

\section{The Area Spectrum}

Henceforth I use the compact notation $\hat{\mathcal{R}}_{\lambda} \equiv \hat{\mathcal{R}}_{n_{\lambda} q_{\lambda} j_{\lambda} m_{\lambda} s_{\lambda}}, D_{\kappa} \equiv D_{n_{\kappa} s_{\kappa}}$ etc. Evidently by closure and linearity

$$
\left[\hat{\mathcal{R}}_{\kappa}, \hat{\mathcal{R}}_{\lambda}\right]=\sum_{\mu} \epsilon_{\kappa \lambda}^{\mu} \hat{\mathcal{R}}_{\mu}+\mathcal{C}_{\kappa \lambda} \hat{I}+\mathcal{D}_{\kappa \lambda} \hat{Q}+\sum_{m} \mathcal{F}_{\kappa \lambda}^{m} \hat{J}_{m}+\mathcal{E}_{\kappa \lambda} \hat{A}
$$

with all structure constants antisymmetric in $\kappa$ and $\lambda$. I assume that for fixed $\kappa \neq \lambda$, not all $\epsilon_{\kappa \lambda}^{\mu}$ vanish (see below). Obviously $|\kappa, \lambda\rangle \equiv\left[\hat{\mathcal{R}}_{\kappa}, \hat{\mathcal{R}}_{\lambda}\right] \mid$ vac $\rangle$ is a superposition 
of basis states $|n q j m s\rangle$, i.e., a one-black hole state. What is special about it ? Commute (23) with $\hat{A}$ to get $\sum_{\mu} \epsilon_{\kappa \lambda}^{\mu} a_{\mu} \hat{\mathcal{R}}_{\mu}$ in the r.h.s. while the Jacobi identity together with Eq. (22) gives $\left(a_{\kappa}+a_{\lambda}\right)\left[\hat{\mathcal{R}}_{\kappa}, \hat{\mathcal{R}}_{\lambda}\right]$ in the l.h.s. Consistency then demands that $\mathcal{C}_{\kappa \lambda}=\mathcal{D}_{\kappa \lambda}=\mathcal{F}_{\kappa \lambda}^{m}=\mathcal{E}_{\kappa \lambda}=0$ as well as $a_{\mu}=a_{\kappa}+a_{\lambda}$ whenever $\epsilon_{\kappa \lambda}^{\mu} \neq 0$. Repeat the exercise with $\hat{Q}$ replacing $\hat{A}$ and discover that $q_{\mu}=q_{\lambda}+q_{\kappa}\left(q_{\mu}\right.$ is, of course, a physical value for a charge) whenever $\epsilon_{\kappa \lambda}^{\mu} \neq 0$. Thus $|\kappa, \lambda\rangle$ is an area eigenstate as well as a charge eigenstate (it satisfies the charge superselection rule). Therefore, sums of $a_{n}$ 's belonging to one-black hole states are possible quantum numbers of physical one-black hole states.

The assumption that for fixed $\lambda \neq \kappa$ not all $\epsilon_{\kappa \lambda}^{\mu}$ vanish means $\hat{\mathcal{R}}_{\lambda}$ and $\hat{\mathcal{R}}_{\kappa}$ never commute. Commutativity would signify, as in field theory, that creations of the two black holes are independent processes. Since we know from the classical limit that two black holes can merge into one, this last possibility cannot be a general law, and noncommutativity is the easiest way to introduce the possibility of black hole fusion already at the quantum level.

Operating on $|\mathrm{vac}\rangle$ with the hermitian conjugate of Eq. (22), namely

$$
\left[\hat{A}, \hat{\mathcal{R}}_{\kappa}^{\dagger}\right]=-a_{\kappa} \hat{\mathcal{R}}_{\kappa}^{\dagger}
$$

we discover that $\hat{\mathcal{R}}_{\kappa}^{\dagger}$ must anhilate $\mid$ vac $\rangle$ since $\hat{A}$ is positive definite: $\hat{\mathcal{R}}_{\kappa}^{\dagger}$ has the nature of an anhilation operator. Now commute $\hat{\mathcal{R}}_{\lambda}$ with Eq. (24) and simplify the triple commutator by means of Jacobi's identity to get

$$
\left[A,\left[\hat{\mathcal{R}}_{\kappa}^{\dagger}, \hat{\mathcal{R}}_{\lambda}\right]\right]=\left(a_{\lambda}-a_{\kappa}\right)\left[\hat{\mathcal{R}}_{\kappa}^{\dagger}, \hat{\mathcal{R}}_{\lambda}\right]
$$

It is plain that $|\bar{\kappa}, \lambda\rangle \equiv\left[\hat{\mathcal{R}}_{\kappa}^{\dagger}, \hat{\mathcal{R}}_{\lambda}\right] \mid$ vac $\rangle$, if it is nonvanishing at all, is a one-black hole state because it is obtained from a one-black hole state by attempting to anhilate from it a black hole of different description from the one it actually contains $(|\bar{\kappa}, \lambda\rangle$ is not a linear combination with $|\mathrm{vac}\rangle$ because, as we shall see, it has definite area). Now from Eq. (25) it is clear that $|\bar{\kappa}, \lambda\rangle$ is an eigenstate of $\hat{A}$ with eigenvalue $a_{\lambda}-a_{\kappa}$. Of course this only makes sense when $a_{\kappa}<a_{\lambda}$ because $\hat{A}$ is positive definite. In the opposite case $\left[\hat{\mathcal{R}}_{\kappa}^{\dagger}, \hat{\mathcal{R}}_{\lambda}\right]$ must anhilate $|\mathrm{vac}\rangle$. By replacing $\hat{A}$ in Eqs. (24)-(25) by $\hat{Q}$ one easily shows that $|\bar{\kappa}, \lambda\rangle$ has the definite physically acceptable charge $\left(q_{\lambda}-q_{\kappa}\right)$. We have thus found that positive differences of $a_{n}$ 's belonging to one-black hole states are possible quantum numbers of physical one-black hole states.

A priori the $n$-th (by magnitude) area eigenvalue for a one-black hole state with definite $j, m, q$ should depend on all of these: $a_{n}=a_{n}(j, m, q)$. But the $m$ dependence here is excluded by rotational invariance. Further, combining states labelled by $j_{\lambda}$ and $j_{\kappa}$ should, according to quantum mechanics, give a state with a superposition of angular momenta $\left|j_{\lambda}-j_{\kappa}\right|, \cdots, j_{\lambda}+j_{\kappa}$. But we know that $|\kappa, \lambda\rangle$ and $|\bar{\kappa}, \lambda\rangle$ have definite areas, so it seems - and we shall so assume pending rigorous proof - that $a_{n}$ cannot depend on $j$. We are left with the dependence $a_{n}=a_{n}(q)$. And charge conjugation symmetry tells us that the $a_{n}(q)$ must all be even. 
Obviously $a_{1}(0)+a_{2}(0)$, a possible eigenvalue for zero charge, cannot fall below $a_{3}(0)$, the third eigenvalue. But we cannot allow $a_{1}(0)+a_{2}(0)>a_{3}(0)$, for in that case $a_{1}(0)+a_{2}(0)-a_{3}(0)$ would be a possible zero-charge area eigenvalue falling below the lowest possible one, $a_{1}(0)$ ! Thus $a_{1}(0)+a_{2}(0)=a_{3}(0)$. Similarly one shows that $a_{4}(0)=a_{3}(0)+a_{1}(0)$, and more generally $a_{n+1}(0)=a_{n}(0)+a_{1}(0)$ for $n \geq 2$. Further, $a_{2}(0) \geq 2 a_{1}(0)$ for otherwise $a_{2}(0)-a_{1}(0)$, also an acceptable zero-charge area eigenvalue, would fall below $a_{1}(0)$. But if $a_{2}(0)>2 a_{1}(0)$, then $a_{1}(0)<a_{2}(0)-a_{1}(0)<a_{2}(0)$ so $a_{2}(0)-a_{1}(0)$ would be an eigenvalue in between the first and second ones! Thus $a_{2}(0)=2 a_{1}(0)$. Collecting our results we get for the zero-charge (Schwarzschild) one-black hole area spectrum

$$
\operatorname{Spect}(\hat{A} \mid q=0)=\left\{n a_{1}(0) \mid n=1,2, \cdots\right\}
$$

This shows the promised uniform spacing.

For $q \neq 0, a_{1}(q)+a_{1}(0)$ corresponds to a state of charge $q$, and so it cannot fall below $a_{2}(q)$. Were $a_{1}(q)+a_{1}(0)-a_{2}(q)$ positive, it would be a possible area eigenvalue for zero charge; however, it evidently falls below $a_{1}(0)$ so the mentioned option entails a contradiction. Thus $a_{1}(q)+a_{1}(0)=a_{2}(q)$. Similarly one can show that $a_{n}(q)+a_{1}(0)=a_{n+1}(q)$ for $n \geq 2$. Thus the area spectrum for fixed $q \neq 0$ shows the same uniform spacing as (26), but possibly a different lowest eigenvalue. The question now is, is $a_{1}(q) \neq a_{1}(0)$ ?

Were $a_{1}(q)-a_{1}(0)$ positive, it would be a possible area eigenvalue for charge $q$, so that $a_{1}(q)-a_{1}(0) \geq a_{1}(q)$. However, this would imply that $a_{1}(0)=0$ which is contrary to the basic assumption. One option is that $a_{1}(q)=a_{1}(0)$ in which case the charge $q$ black hole spectrum is identical to that of the neutral one, (26). The other is that $a_{1}(0)-a_{1}(q)$ is positive and so is a possible area eigenvalue for charge $-q$. But $a_{n}(q)$ is even so we have $a_{1}(0)-a_{1}(q) \geq a_{1}(q)$, or $a_{1}(0) \geq 2 a_{1}(q)$. Further, $a_{1}(q)+a_{1}(-q)$ is evidently a possible area eigenvalue for zero charge. It follows that $2 a_{1}(q) \geq a_{1}(0)$. This is consistent with our previous finding only if $a_{1}(q)=\frac{1}{2} a_{1}(0)$, which constitutes the second option.

I now show that for $q \geq 2$ the second option is possible only if already $a_{1}(1)=$ $\frac{1}{2} a_{1}(0)$. First suppose that for some $\tilde{q} \geq 1, a_{1}(\tilde{q}) \neq a_{1}(\tilde{q}+1)$. Then by the last paragraph $\left|a_{1}(\tilde{q})-a_{1}(\tilde{q}+1)\right|=\frac{1}{2} a_{1}(1)$. On the other hand either $a_{1}(\tilde{q})-a_{1}(\tilde{q}+1)$ stands for a state of type $|\bar{\kappa}, \lambda\rangle$ with charge -1 or $a_{1}(\tilde{q}+1)-a_{1}(\tilde{q})$ stands for one of charge +1 . In either case $\frac{1}{2} a_{1}(1)=\left|a_{1}(\tilde{q})-a_{1}(\tilde{q}+1)\right| \geq a_{1}(1)$. But this is inconsistent if $a_{1}(1)=a_{1}(0)$. Thus if $a_{1}(1)=a_{1}(0), a_{1}(q)=a_{1}(q+1)$ also for all $q \geq 1$ and the spectrum of $\hat{A}$ for all $q>1$ is just like that for $q=0$, namely (26).

For the alternative $a_{1}(1)=\frac{1}{2} a_{1}(0)$ we can also have cases with $a_{1}(q)=\frac{1}{2} a_{1}(0)$, $q>1$, and the spectrum is

$$
\operatorname{Spect}(\hat{A} \mid q \neq 0)=\left\{(n-1 / 2) a_{1}(0) \mid n=1,2, \cdots\right\}
$$

for all these. How do these conclusions square with Barvinsky, Das and Kunstatter's [7] conclusion from canonical quantum gravity that $a_{1}(q)$ goes up like $q^{2}$ ? The 
apparent contradiction may reflect our omission from the algebra of some operator, on the par with $\hat{A}, \hat{Q}$ and $\hat{\mathbf{J}}$, which does not commute with all of them. Then some of the eigenvalues of $\hat{A}$ we have found with our sparser subalgebra may be "killed" by consistency requirements. In fact, this may be necessary because according to Eq. (11), in the classical limit $A \geq 4 \pi Q^{2}$, so that area eigenvalues for large $q$ may simply have to be large even for $n=1$.

\section{The Degeneracy Factor and Black Hole Entropy}

As mentioned in Sec. 3, the area eigenvalue corresponding to state $\mid$ nqjms $\rangle$ may be degenerate to the tune of $g_{n}$. By the same symmetry arguments we applied in Sec. (4) to conclude that $a_{n}=a_{n}(q)$ only, we conclude that $g_{n}=g_{n}(j, q)$ only.

Consider now the $g_{n_{\kappa}}$ different states $\left|n_{\kappa}, j_{\kappa}, m_{\kappa}, q_{\kappa}, s\right\rangle$; we assume not both $j_{\kappa}$ and $q_{\kappa}$ vanish. And consider also the $g_{1}$ different states $\left|n_{\lambda}=1, j_{\lambda}=0, m_{\lambda}=0, q_{\lambda}=0, s\right\rangle$. We can form $g_{1}(0,0) \cdot g_{n_{\kappa}}\left(j_{\kappa}, q_{\kappa}\right)$ states $|\kappa, \lambda\rangle$ in the notation of Sec. 1 , which we shall suppose to be independent. According to our results in Sec. 4 , all the $|\kappa, \lambda\rangle$ have charge $q_{\kappa}$. Further, since we are combining zero angular momentum with $j_{\kappa}$, it seems obvious (and can be established rigorously by use of Eqs. (20)-(21)) that all the $|\kappa, \lambda\rangle$ bear angular momentum $j_{\kappa}$. By Sec. 4 all our $|\kappa, \lambda\rangle$ here have area quantum number $n_{\kappa}+1$. Since there are a total of $g_{n_{\kappa}+1}\left(j_{\kappa}, q_{\kappa}\right)$ one-black hole states with these quantum numbers we must have

$$
g_{1}(0,0) \cdot g_{n_{\kappa}}\left(j_{\kappa}, q_{\kappa}\right) \leq g_{n_{\kappa}+1}\left(j_{\kappa}, q_{\kappa}\right)
$$

Henceforth we drop the subindex $\kappa$.

One immediate consequence of Eq. (28), given that $g_{1}(0,0) \geq 1$, is that $g_{n+1}(j, q) \geq$ $g_{n}(j, q)$ : degeneracy cannot decrease with $n$. Let us iterate (28) starting from $n_{\kappa}=1$ and assuming $g_{1}(0,0) \neq 1$ :

$$
g_{n}(j, q) \geq g_{1}(j, q) \cdot g_{1}(0,0)^{n-1}
$$

Thus $g_{n}(j, q)$ grows at least exponentially with $n$. We can think of $\ln g_{n}(j, q)$ as the entropy associated with a black hole with quantum numbers $j, m$ and $q$ simply because the degeneracy represents a multiplicity of states which are observationally indistinguishable. Taking the logarithm and comparing with Eqs. (26)-(27) shows that the entropy must grow at least as fast as the area. If it grows exactly as area we get the usual black hole entropy formula $S \sim A$ in the classical limit. The proportionality factor, $\ln g_{1}(0,0) / a_{1}(0)$, must equal the $(4 \hbar)^{-1}$ of Hawking's formula. Adopting $g_{1}(0,0)=2$ for illustration we deduce [13]

$$
a_{1}(0)=4 \hbar \ln 2
$$

which calibrates the area spectrum (26)-(27). The discrete mass spectrum (5) then follows from Eq. (画). 
Another possibility is that entropy grows somewhat faster than area. For example, $g_{n}(j, q) \sim n g_{1}(0,0)^{n-1}$. In this case we get a correction like $\ln A$ (with positive coefficient) to the entropy. This comes directly from the degeneracy, not from quantum fluctuations, etc.

Acknowledgement: I thank Jim Hartle, John Schwartz and Gilad Gour for incisive remarks. This research is supported by grant No. 129/00-1 of the Israel Science Foundation.

\section{References}

[1] C. M. Misner, K. S. Thorne and J. A. Wheeler, Gravitation (Freeman, San Francisco 1973), pp. 872-915.

[2] S. W. Hawking, Phys. Rev. D 26, 1344 (1971).

[3] J. D. Bekenstein in Black Holes, Gravitational Radiation and the Universe, eds. B. R. Iyer and B. Bhawal (Kluwer, Dordrecht 1999).

[4] A. E Mayo, Phys. Rev. D 58, 104007 (1998).

[5] J. D. Bekenstein, Phys. Rev. D 7, 2333 (1973).

[6] M. W. Duez, et al., Phys. Rev. D 60, 104024 (1999).

[7] A. Barvinsky, S. Das and G. Kunstatter, "Spectrum of charged black holes - The big fix mechanism revisited", gr-qc/0012066 and "Quantum mechanics of charged black holes", hep-th/0102061.

[8] J. D. Bekenstein in Advances in the Interplay between Quantum and Classical Physics, ed. V. de Sabbata (Kluwer, Dordrecht 2002).

[9] M. Born, Atomic Physics (Blackie, London, 1969), 8th edition.

[10] D. Christodoulou and R. Ruffini Phys. Rev. D 4, 3552 (1971).

[11] J. D. Bekenstein, Lett. al Nuovo Cimento 11, 467 (1974).

[12] V. Mukhanov, JETP Letters 44, 66 (1986).

[13] J. D. Bekenstein and V. F. Mukhanov, Phys. Lett. B 360, 7 (1995).

[14] M. Schiffer "Black hole spectroscopy", preprint IFT/P-38/89, São Paulo (1989); Y. Peleg, Phys. Lett. B 356, 462 (1995); H. Kastrup, Phys. Lett. B 385, 75 (1996); J. Louko and J. Mäkelä, Phys. Rev. D 54, 4982 (1996); A. Barvinsky and G. Kunstatter, Phys. Lett. B 329, 231 (1996); V. A. Berezin, Nucl. Phys. Proc. Suppl. 57, 181 (1997); C. Vaz and L. Witten, Phys. Rev. D 60, 024009 (1999); C. Vaz, Phys. Rev. D 61, 064017 (2000). 
[15] J. Mäkelä and P. Repo, Phys. Rev. D 57, 4899 (1997); C. Vaz and L. Witten, Phys. Rev. D 63, 024008 (2001); J. Mäkelä, P. Repo, M. Luomajoki and J. Piilonen, Phys. Rev. D 64, 024018 (2001).

[16] A. D. Dolgov and I. B. Khriplovich, Phys. Lett. B 400, 12 (1997); V. A. Berezin, Phys. Rev. D 55, 2139 (1997); V. A. Berezin, A. M. Boyarsky and A. Yu. Neronov, Phys. Rev. D 57, 1118 (1998).

[17] A. Ashtekar and J. Lewandowski, Class. Quant. Grav. 14, A55 (1997).

[18] A. Alekseev, A. P. Polychronakos and M. Smedbäck, "On area and entropy of a black hole", hep-th/0004036; I. B. Khriplovich, "Quantization and entropy of black holes", gr-qc/0106012.

[19] G. Gour, Phys. Rev. D 61, 12400 (2000).

[20] E. Merzbacher, Quantum Mechanics (Wiley, New York 1970), 2nd edition, pp. 396-404. 